\title{
Design and Implementation of a Witness Unit for Opportunistic Routing in Tsunami Alert Scenarios
}

\begin{abstract}
Some earthquakes generate tsunamis in the ocean or coastal regions. An early alert is essential for the coastal population to prevent loss of human life. The propagation of the alert is made through a range of communication means: radio, television, sirens, landlines and cell phones, social media including Twitter, and, if electrical power and communications utilities are blacked-out, through an alternative opportunistic network. In this article, a special device, named Witness Unit (WU), designed to work with a satellite network is presented as an alternative communications means in a temporarily degraded disaster environment. A greedy heuristic algorithm for the placement and deployment of the WUs is introduced and its interaction with emergency managers and citizens is discussed.
\end{abstract}

Keywords: Tsunami alert, Opportunistic Networks, Evacuation

\section{Introduction}

Tsunamis are one of the many consequences that earthquakes in the ocean or coastal regions may cause. If an earthquake generates a tsunami, the population in seaside towns and cities should be evacuated to prevent human injury and life loss. An early alert of the situation is very important as the probability of survival is strongly linked to the chance of people reaching a safe place or shelter. However, if the earthquake affects the city's infrastructure, producing power utility and communication failures, the coordination of the evacuation procedure with little to no communication becomes one of the most challenging issues to be solved. An early detection and alert system for near-field tsunamis has several components, as presented in other articles in this special issue. This article focuses on the implementation of an alternative communication network that can dynamically provide the necessary 
information for the population to reach shelter in case an earthquake causes a tsunami and the collapse of traditional communication networks.

The proposed information retrieval and dissemination method is based on a dynamic and interactive design, operating at several levels of authority and information exchange. The Emergency Operations Center (EOC) is responsible for alerting the population to tsunami risk and coordinating the evacuation procedure. Officials at the EOC transmit the tsunami alert through multiple independent methods like TV, radio, sirens, social media, and street signs. Community leaders, such as Imams at mosques or principals at schools, are used to guide groups of local residents or students in their immediate care to safety. People tend to trust familiar leaders more than formal announcements, and the goal is to harness existing forms of social organization to mobilize collective action rapidly. The local leaders seek to guide their followers to a safe place or shelter, based on information they receive from reliable sources. Such information is provided by the EOC through any functioning means of communication or through our proposed alternative opportunistic network (OppNet), explained in more detail below. The leaders may also contribute to the state-of-affairs by providing updated information to the decision support system. For example, local leaders may indicate that a route is blocked and cannot be used any more or update the status of congestion on certain routes. There is a very short time between the seismic alert and the arrival of the tsunami, so the decision support system operates within hard, real-time constraints (Stankovic, 1988). The proposed system is based on GIS layered maps so local information is stored in the leaders' smartphones, reducing the data traffic in the opportunistic network (Ai et al., 2015, this issue).

In the last decade, the use of social networks has changed completely the way in which people interact. There are several examples of people using, for example, Twitter to self-organize rescue procedures after natural disasters like floods (Meier, 2015). These uncoordinated efforts were helpful in some situations and show the potential of these tools. The use of information and communication technologies (ICTs) during emergencies should improve the resilience of the overall system, that is, the ability of organizations to adapt to unexpected situations that may turn into catastrophe (Wybo and Lonka, 2002). In the case of our proposed system, the ICT support is focused on assisting community leaders in the decision process, in particular in the search of secure routes to shelters. This has been identified in previous events as one of the main tsunami safety issues. The introduction of an alternative network 
to ensure communication flow between people and emergency managers is of major importance in scenarios in which traditional communication means and power utility are unavailable. The use of this network requires special training for the community leaders and the general population to take advantage of the technology during the critical interval of time between the tsunami alert and the actual wave arrival.

The deployment of an alternative opportunistic network is one means of communication that may augment the range of methods needed for issuing early warning for an imminent tsunami. Its deployment also provides support for the after-event search and rescue process. Once the earthquake has subsided and the tsunami has washed over the coastline, there are only a few hours to rescue survivors. This period is called the golden relief time and lasts approximately 72 hours (Ochoa and Santos, 2015). After a devastating tsunami event, there is likely very little functional infrastructure, so deploying an alternative network to guide search and rescue efforts is very important.

This article makes three contributions. First, we present the design and a scenario for the implementation of a witness unit (WU) to be used as a communication gateway between the community leaders and the EOC. These WUs are built with off the shelf (COTS) components; they are cheap, easily assembled, and provide the necessary functionality to route messages between personnel at the EOC and the community leaders. Second, we devise a heuristic algorithm to bypass the NP-Hard coverage deployment problem of the WUs throughout the city. Finally, we propose an information dissemination policy to inform people of the best ${ }^{1}$ route to a shelter from their existing location. This communication process is interactive and dynamic.

The paper is organized as follows. Section 2 describes the main elements used to build the WU and its functionality. Section 3 outlines the implementation steps for deploying WUs, providing technical information. Section 4 describes a heuristic deployment algorithm for the WU network throughout the city. Section 5 presents the opportunistic dissemination policy. In Section 6 the information flow among the different agents (EOC, leader, community) is presented. In Section 7, the WU functionalities are discussed, extending

\footnotetext{
${ }^{1}$ Note that "best" is typically decided based on speed of evacuation time but can also take into account the ability of people to climb stairs, use bicycles, etc. These factors are outside the scope of this paper.
} 
the application of this technology to other areas. In Section 8 previous work in the area is reviewed, and conclusions are drawn in Section 9.

\section{Materials and Methods for a WU Network}

The basic elements needed to build a WU are explained and a functional description of each part is introduced without proposing any commercial

solutions. Assembly and software implementation details are provided in Section 3.

In situations where communication is needed but traditional Internet or cell infrastructures fail to provide it effectively, the best alternative may be opportunistic networks, which are characterized by intermittent connectivity, a heterogeneous mix of nodes, node churn, and widely varying network conditions. The purpose of WUs is not to provide an alternative network for everyday communications. Instead, the use of these units should be triggered only during emergency situations in which traditional networks are down or have collapsed. After an earthquake or any large disaster, electricity is typically either disrupted or shut down to prevent fires. If the earthquake also affects telephone lines and telecommunications infrastructure, most daily means of communication are blacked out and what little communication resources remain working are usually overloaded and become unusable. It is in this degraded technical context that the use of an OppNet becomes a feasible alternative. The OppNet can be defined as a peer-to-peer application in which nodes implement a basic store-and-forward transfer protocol. However, given that OppNets are not reliable, we add a WU, with embedded system design characteristics, to provide connectivity from nodes to the leader's smartphones (the main device used in many situations, and a device that will probably replace the currently ubiquitous walkie-talkie) in disaster situations.

Our proposed WUs are built based on three main components: a small on-board computer, a satellite modem, and a Wi-Fi access point device. The leaders will have in their smartphones an application that can connect to the WU access point via Wi-Fi to receive and send information. The leader will receive relevant information on how to reach the nearest shelter following the quickest route; this is possible because the WUs are static and the leaders have geo-localization (i.e., GPS capabilities and the supporting software ). At the same time, the application on the leader's smartphone will upload real-time information it can sense, such as streets blocked by fallen trees or 


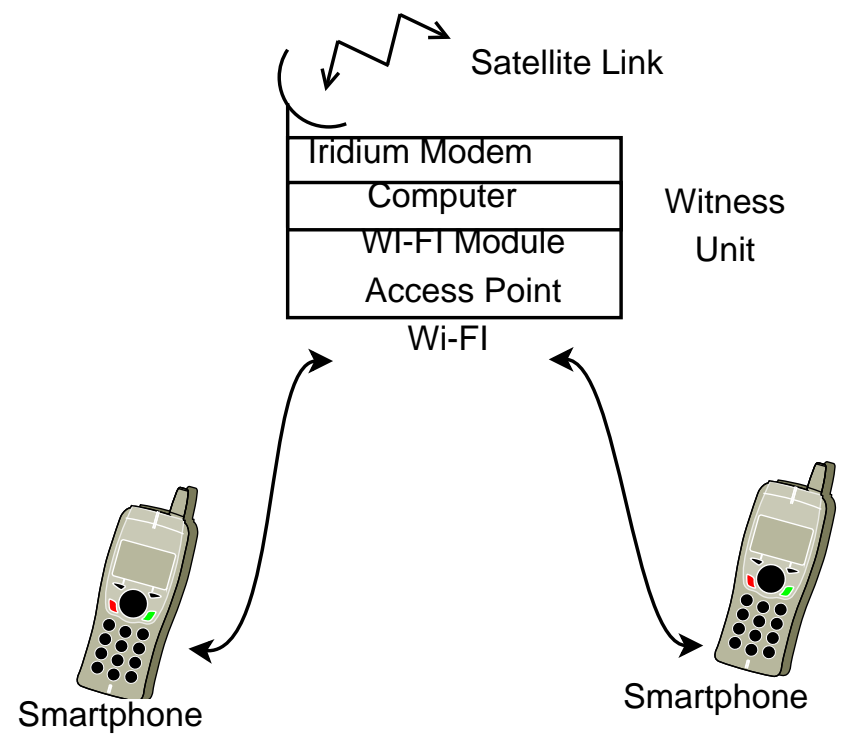

Figure 1: Witness Unit General Schematic

debris, broken bridges, etc. (Ai et al., 2015, this issue). This information is used by the EOC to update the situation and provide safe routes to leaders. Figure 1 shows the architecture of the WU and its interaction with leaders.

\subsection{Access to Witness Units}

The most accepted protocol today for wireless communications is based on the IEEE 802.11 standard, (IEEE, 2010). In addition to having a Wi-Fi radio, even cheap smartphones are sold with a GPS device, and the leaders will certainly have smartphones. The WU will provide an ad-hoc Wi-Fi interface access point (AP) that is easily identified by the Service Set Identifier name (SSID), called WUAP-address, where address indicates the location of the WU. The AP will be accessible through a simple association process provided in the application executing in the leader's smartphone. WU access is restricted to certified leaders as its purpose is to provide information before, during, and after disasters (e.g., earthquake and tsunami alerts) and not to provide Internet access on a regular basis. As the smartphone of each leader is geo-located by its GPS and the locations of the WUs are known, the application will easily find the relevant WU in each area, and quickly associate with it. 


\subsection{On-board computer}

The WU is not a simple AP. It should have the capability of executing the basic opportunistic transfer protocol of store-and-forward (Cao and Sun, 2013). The WU will receive the alerts from the EOC and will also receive updates from the leaders, such as new information on blocked or congested roads. This information would be updated in the EOC through the satellite connection, but it will also be kept at the WU because it may be useful for other leaders getting in contact with that WU. There are many on-board computers available in the market with enough capabilities for a WU as mentioned in Section 3. As these units do not use hard disks or monitors for displaying information and do not require a keyboard, they can be assembled in a small device working on batteries for an extended period of time. Such a feature is critical, especially because the WU is designed for scenarios where electricity has been shut down or access to utilities has been severed.

\subsection{Satellite Modem}

Clearly, installing a new satellite network would be prohibitively expensive. In our design, we propose the use of Iridium satellites to provide the connectivity needed for disaster management. Iridium was an ambitious project designed to provide satellite communications through cell phones all over the world. A complete constellation of low orbit satellites that have been launched into space is scheduled to continue working at least until 2020. Although the general project of satellite telephones was never completed, the constellations of satellites still provide an active data network through special modems and the Short Burst Data (SBD) protocol (Iridium, 2012).

\section{Implementation}

This section presents a technical description of WUs, based on the three components described above in Section 2, the interface among the components, and the software used to operate them.

\subsection{Technical details}

\subsubsection{On-board Computer}

For ease of assembly, maintenance and acquisition, the WU is built upon well-known and well-recognized commercial devices. These devices have been

used in different applications and have a large community of developers (both 
free/open source and commercial) that provide technical support. Alternatively the WU could be designed and built from scratch. Although it could potentially be cheaper for the hardware, it would take time to build and customize the WU, and it is unlikely it would have extra functionalities compared to those already available.

The core unit should have the capability of running an operating system, do basic network routing, receive, store and process information related to the tsunami alert and the search and rescue procedure. To implement these functionalities, the board should have a serial interface to drive a modem and the possibility of connecting to $\mathrm{Wi}-\mathrm{Fi}$ modules. The concept is that the WU can replicate the relevant data provided by the central EOC server, so it is a requirement that the WU have the capacity to connect to external memory such as Secure Digital (SD) or micro SD card memories. As explained in more detail in Section 7, the WU can be used as a sensor unit. For this, it should have the possibility of being connected to different sensors like weather sensors (temperature, wind and humidity), accelerometers, noise, and even video cameras. There are several development boards with these capabilities like Arduino Intel Galileo (Intel, 2015), Raspberry Pi (Raspberry, 2015) or BeagleBone (Coley, 2015).

\subsubsection{Wireless ad-hoc network}

The WU provides an AP for community leaders. There are several wireless protocols that can be used in OppNets such as Bluetooth (Bluetooth, 2014) or ZigBee (ZigBee, 2015) but they are limited in communication range and number of simultaneous connections. Wi-Fi is the common name of the IEEE 802.11 standard (IEEE, 2010). Every smartphone in the market has the capability of connecting to a Wi-Fi AP. There are several IEEE $802.11 \mathrm{~b} / \mathrm{g} / \mathrm{n}$ modules available in the market that can be used off the shelf to convert the on-board computer to a Wi-Fi router AP. Today, most Wi-Fi implementations work with the $802.11 n$, which has at least a 100 -meter communication range. Due to the potential lack of connectivity, the evacuation/routing decision to the nearest shelter is typically based only on local data and not on global data. With our design, the WU and the application succeed in getting access to the EOC to obtain regular updates on shelter occupancy and evacuation routes to nearest available shelters, thus potentially reducing casualties. The appropriate (local/regional) information can be updated to the appropriate WUs continuously, so the leader only needs to get in contact with one WU to receive the latest information. 


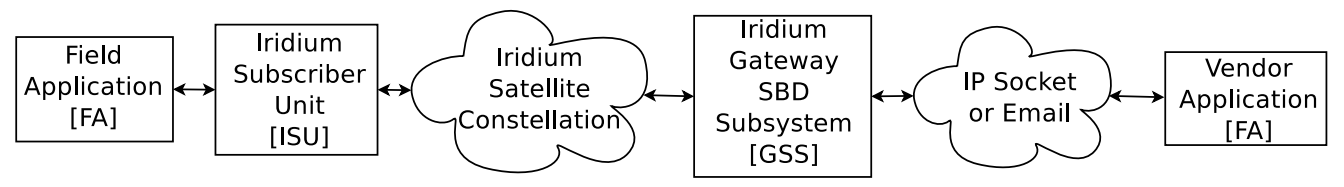

Figure 2: Iridium System (source: Iridium, 2015)

The access to this Wi-Fi network is restricted and only people/devices with the appropriate credentials can gain access to send messages to the EOC, while anyone can receive broadcast information from the WU. Access is provided through a special application developed in the mobile platform. Once in range of one of the $\mathrm{WU}$, the application will connect to it and will immediately begin to exchange information. As proposed in (Ai et al., 2015, this issue) the application is designed to have pre-loaded maps and shelter locations.

\subsubsection{Satellite Connectivity}

Earthquakes may have a devastating impact on the built environment and critical infrastructure in cities, destroying facilities even before the tsunami arrives. Iridiums Short Burst Data Service (SBD) (Iridium, 2012) is a simple and efficient satellite network transport capability to transmit short data messages between field equipment and a centralized host computing system. Figure 2 shows the basic components in the transmission process. Messages are transported using either an e-mail client/server structure or through DirectIp (Iridium, 2012). Two kinds of messages, mobile originated and mobile terminated, are defined depending on whether they are sent or received by the field device, respectively.

The Iridium subscriber unit (satellite modem) provides an SBD interface to the Iridium constellation. The WU software would route the messages from the community leaders to the EOC and from the EOC to the community leaders. Thus, the WU would provide continuous up-to-date information to all users in its range on the best and quickest escape routes to shelters and safe places. The information would flow in both directions, and given the rare occurrences of tsunamis and earthquakes, the best design is that of a push-pull or pub-sub protocol, to reduce the number of messages that would flow through the WU. Furthermore, since the on-board computer can be easily configured with a large amount of memory, data can be stored there 
for later release to the leaders or followers who, for whatever reason, can only access the information at a later stage. In this way, each WU has a copy of the latest data generated by the EOC and can provide fast guidance to the leaders. The WU also keeps a log of all the EOC-WU data traffic, and can run local algorithms to optimize the evacuation based on local requests (from local leaders who are connected).

When a message from one leader connected to the WU network is processed, the WU decides if this message contains new or dated information. New information provided by the application (e.g., about problems on the different routes) is immediately uploaded to the server via the satellite network. The only traffic using satellite communication is the two-way flow of information between the EOC and the WU.

\subsection{Software}

We can identify two different sets of software subsystems that are equally important. The first subsystem is made up of the hardware drivers and libraries needed to make the hardware system work. Examples of these are the libraries for the access point definition and handling, the library for the Iridium SBD modem, and the library for handling the SD memory. These tools are necessary to put the system in operation. The second consists of several applications, including the application on the mobile device, on the WU, and on the EOC server, all of which are developed to provide information retrieval and dissemination. This is the functional part of the WU, which accounts for the communication between the WU and the smartphone leader application, and for the communication between the WU and the EOC server. In both cases, we choose the Client-Server paradigm given the communication patterns that arise between leader-WU and WU-EOC server. Note that the WU is a server in the first case and a client in the second. Details of these software components are described below.

\subsubsection{WU-Leader communication}

As explained above, the $\mathrm{WU}$ is in constant communication with the EOC server at least every $t$ time units (e.g., a minute). The WU should have enough available memory to store the GIS information necessary to build a map, identify the shelters and their occupancy, availability of hospitals, street and avenue conditions, in order to determine the best routes to safety. The processing capacity of the WU is sufficient for identifying the nearest shelter and best route to it. This can be precomputed by the EOC server 
based on global information or it can be computed (or enhanced) based on the local information from the local leaders. Once a leader has connected to the WU, it sends a message identifying him/herself and asking for the nearest shelter with enough vacancy for his/her followers. The message uses the smartphones GPS to track the leader. The information transmitted has the following format, according to (Ai et al., 2015, this issue):

$$
\text { Shelter AllocReq }=<\text { timestamp, leaderID, lat,long, followers }>
$$

where timestamp is the time at which the message is issued, leaderID is the leaders identification, obtained automatically from the smartphone, lat and long refer to the latitude and longitude from the leaders GPS, and followers contains the number of people the leader represents.

After receiving this message, the $\mathrm{WU}$ decides if it can provide the response immediately (i.e., it has up to date information) or has to ask the EOC server. In Figure 3, Algorithm 1 shows the procedure, which considers the state of the data according to the last update to the WU. Being updated within $t$ minutes is considered as "updated".

The WU returns to the leader the ShelterAllocRoute message identifying the time at which it is produced, the WU providing it, the shelter identification and the route. The message structure is as follows:

$$
\text { Shelter AllocRoute }=<\text { timestamp, WUid, shelterid, route }>
$$

Result: Shelter selection and route

1. Check last update time, TIME

2. If (timestamp-TIME $>t$ AND Satellite Connectivity is available)

(a) Call EOC client service.

(b) Update local database.

3. Search for nearest best shelter/capacity.

4. Determine route to shelter.

5. Return ShelterAllocRoute

Figure 3: Algorithm 1. Shelter Allocation Procedure in the WU

The route field in the message is composed by a sequence of streets information and landmarks. Each WU may have a pre-loaded set of routes for 
Data: RouteStateInfo, Routes Allocation Table

Result: Obstacles in routes

1. Update local Route Allocation Table with timestamp.

2. Send RouteStateInfo message to EOC server.

Figure 4: Algorithm 2: Routes State Update Algorithm

the different shelters and can remove them from the list as they are being declared disrupted by the leaders or by the EOC. If the pre-loaded routes have all been classified as not usable, an alternative new route has to be computed.

Leaders may report problems to the EOC through the WU. For this, a RouteStateInfo message is generated and transmitted to the WU. The message has the following format:

RouteStateIn $f_{o}=<$ timestamp, leaderid, latObst, longObst, obstacle $>$

Leaders mark the obstacles in the application by using an easy-to-create event as they come across them (e.g., a bridge is down 100 meters north of my location) or pointing and clicking on a map. However, these events, which become messages, are only transmitted to the WU/EOC once the leader is in communication with a WU. Therefore, while the ShelterAllocReq message is generated by the leaders, the RouteStateInfo is sent automatically by the application as they get connected with (i.e., in range of) the WU. In Figure 4, Algorithm 2 shows the procedure.

\subsubsection{EOC-WU communication}

The communication between the WU and the EOC is made through the SBD (Short Burst Data) protocol, as explained above. The Iridium SBD library uses the RockBLOCK/Iridium's SBD protocol to send and receive short messages to/from the Iridium hub (Hart, 2014). SBD is essentially a text message, and can be described as a technology that supports the transmission of text or binary messages up to a certain maximum size (270 bytes received, 340 bytes transmitted). In this section we explain how we integrated the Send and Receive methods with the WU to be able to: (a) inform the EOC of changes in the regional/local route states, (b) to obtain an updated version of the shelters' accommodation capacity, and (c) to obtain the state of the streets informed by other WUs (i.e., events). In this way, the 


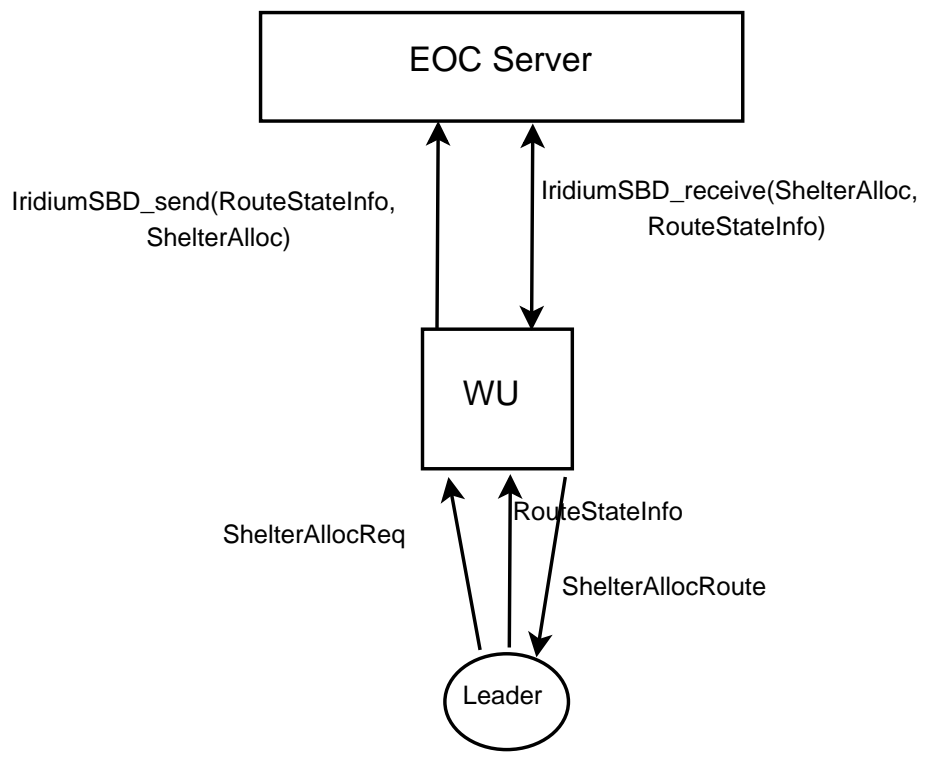

Figure 5: Message Exchange Model

WU can have an updated copy of the information provided by the EOC and other WUs, and is able to compute the best local shelter and route to it for a particular leader and his followers.

Every $t$ time units the WU should request an updated copy of the information. Only the changes are informed by the EOC server. For example, if shelter A has received people (or is scheduled to receive people) and thus has reduced capacity, only the data pertaining to the changes is returned to the WU (in this case, specifying shelter A's new capacity or reduction thereof). Before updating the routes to the different shelters, the WU will send the RouteStateInfo received from the leaders, and only then will the WU retrieve the new routes. This way, the last version is always present in the WU and transmitted to the leaders. Figure 5 shows the communication model and message exchange.

\section{WU Deployment}

The use of the WUs as alternative gateways for communication between the community leaders and the EOC requires a proper deployment of the units in the area of interest. However, WU networks are akin to a special 
wireless sensor network with particular constraints that should be considered. In this section we address three questions: How many WUs are needed for coverage? Where should the WUs be located? And how much redundancy is necessary to ensure that the messages are actually received by the leaders and the EOC?

The distribution of WUs throughout the city should take into account the actual distribution of the population, the predetermined routes to the different shelters, and the vulnerability of the area to both the earthquake and the tsunami. With these variables, WUs should be deployed to provide the community leaders, and through them the general population, with reliable, up-to-date information during the evacuation procedures. To achieve this objective, it is not necessary to provide continuous access to a $\mathrm{WU}$, as it is intended that the WU would deliver updated information regularly.

Given that it is essential for WUs to survive earthquakes and, if possible, tsunamis, the appropriate location of the WUs needs to be determined. By examining hundreds of photographs of post-earthquake areas, we have observed that traffic lights and electricity poles are systematically found to be standing; this is probably due to the simple structure and low mass and resistance of these structures. Therefore, we choose to install the WUs in traffic lights or utility poles. Furthermore, it is fortuitous that traffic lights and utility poles tend to be placed at road crossings typically away from buildings. They are also usually well cemented and stand a relatively good chance of not being buried by collapsing buildings. Those poles with WUs could also be equipped with signs indicating to the community leaders the presence of a WU. WUs are cheap and can be packaged in a small box that can be easily attached to the poles. They are small and would therefore not catch the attention of passersby, reducing vandalism and theft. WUs may work on electricity, solar energy, or batteries, which can be provisioned to last as many hours as required (if there is a solar panel, the energy concerns might be much smaller). Clearly, solar panels may increase the cost of the system and potentially make it more attractive to occasional thieves.

The deployment of WUs in urban areas requires consideration of factors outlined above that may facilitate local coverage. Below we propose an analytical method to determine the location of WUs, the preferred degree of redundancy, and the total number of units to be deployed.

WUs should ideally be placed at road crossings. This is not just because traffic lights and utility poles are likely to withstand earthquakes and tsunamis, as explained above, but also because people are evacuated along 
roads, and so WUs at road intersections would be practical. Accordingly, we impose a restriction that WUs are placed on roads, preferably at road crossings. Another consideration is that predetermined escape routes should have a virtual breadcrumb trace along them, so the community leaders and their followers can be informed of any relevant changes as soon as possible. The optimal distance between two successive WUs along the escape route depends on the way in which people actually move to the shelters. Given that tsunami alerts have a very short time span, people should move very quickly to reach the shelter. For the design of an algorithm we assume that the distance between any two successive WUs should be at most $D$. Thus, an escape route of length equal to $10 D$ should have at least 9 WUs separated by $D$ meters. The distance can be computed from the time needed by a person to move from one WU to the next. Clearly, $D$ will vary with the type of transportation used and whether the route is a straight line between WUs.

As modeled in most computer systems, a city street map can be seen as a graph in which every street intersection is a node and the block between two intersections is an edge. In this way the city can be represented by $G(V, \xi)$, where $V$ is the set of nodes and $\xi$ is the set of edges linking them. Each edge has an associated weight that indicates the distance in meters between the nodes. To avoid working with a huge graph, the city can be divided into smaller regions, $R_{i}$, such that any two regions are independent, that is $R_{i} \cap R_{j}=\Phi$ for every $i, j$. Regions are defined based on demographic or socio-technical information provided by the local authorities; their definition is beyond the scope of this paper. Every region should have one or more shelters for the population living or working in it, and should also have predefined routes to reach them. Routes also should be available to reach alternative shelters out of the region. In any case, these predefined routes provide the first deployment locations for the WUs. Thus, the problem consists in identifying the intersections that should be used to provide coverage to the region, so people who are actually not on the route to the shelter can reach it and be evacuated.

The distribution of the WUs is a typical coverage problem and has been proved to be NP-Hard (Huang and Tseng, 2005). As there is no known polynomial solution, we propose a heuristic approach based on a greedy algorithm. The main idea is that, starting at a pre-defined WU, the graph corresponding to a region is traversed. The edge with the highest associated weight is followed at each step until the sum of the traversed edges is bigger than the predefined $D$. The immediate previous node traversed is tenta- 
tively used as a WU holder. The procedure is repeated until all the edges connected to the original vertex have been followed. The same algorithm is done for each node holding a WU. In Appendix Appendix A the algorithm is formalized, its complexity is computed and an example is provided for the interested reader.

\section{Opportunistic Information Dissemination}

The use of social networks, and in particular Twitter, was proposed as the main communication link between the EOC, the leaders and the followers (Carley et al., 2015, this issue). In this section we discuss the opportunistic dissemination of information to the community. It is important to recall that in the context of the project, the proposed opportunistic network (OppNet) acts as a backup communication infrastructure and not the main one. There is extensive academic literature addressing different dissemination policies in OppNets. Some papers rely on some kind of infrastructure, either fixed or mobile (Huang et al., 2008), (Liu et al., 2011) and others are only based on the mobility of the nodes (Nguyen and Giordano, 2009). OppNets are defined at the application layer and can use any link and physical layer to transmit a message. That means that a message may be received through a Bluetooth link and forwarded through a Wi-Fi interface. As we intend to provide the general population with an instrument to receive information about the ongoing evacuation procedure, the use of smartphones is a reasonable choice. Smartphones are rapidly becoming cheaper and are very quickly replacing older mobile phones. In particular, the use of mobile phones in Indonesia is very high and is the best option to disseminate information, either because the users have access to social networks like Twitter (primary information channel) or because they can receive the messages from the opportunistic network (Carley et al., 2015, this issue).

The top three smartphone (namely, IOS/iPhone, Android, and Windows) all have an open marketplace where applications are available for download. Smartphones have the capability of communicating through the cell-phone network (G2, G3 or G4) as well as the possibility of connecting through Wi-Fi. Most smartphones also have a Bluetooth interface. Although it is possible to develop an opportunistic application to work in an ad-hoc Wi-Fi mesh network, phones usually have the access to the Wi-Fi interface blocked for use in mesh-network mode. To be able to use the Wi-Fi interface in this mode, a process named rooting in the phone has to be performed and this 
is not an easy task for the layman (Busanelli et al., 2013). For this reason, the opportunistic dissemination policy is not based on $\mathrm{Wi}-\mathrm{Fi}$, but whenever possible, it is based on the Bluetooth ad-hoc mesh network when using smartphones. Leaders and followers, if close enough, may exchange information directly using the Bluetooth interface (Moreira and Milheiro Mendes, 2012). However, this technology requires a very short distance between both nodes; in fact, different Bluetooth standards allow for a trade-off between power consumption and reach of the Bluetooth messages (1 meter consumes 1 milli Watts, 10 meters for 2.5 milli Watts, 100 meters for 100 milli Watts (Bluetooth, 2014)), which can be explored in a future work.

In summary, the sections above explained that the basic opportunistic dissemination policy is based on the principle of store and forward because we cannot assume there is a stable link between two nodes on the network. For this reason, WUs are deployed throughout the city according to the heuristic algorithm presented in Section 4. WUs are critical in the transmission of information, as they become infostations (Galluccio et al., 2010). They receive information both from the leaders and the EOC. Only the leaders are able to send information through the WUs to followers or the EOC, but everyone can receive a message broadcast by a WU as they get into the WU transmission radio. All WUs receive the latest updates about all shelters and the state of the routes periodically and they can broadcast this information to anyone requesting it.

\section{The WU as key elements in the emergency procedure}

We have presented an alternative communication network that becomes active in the case of tsunami alerts. The short interval of time between the issuing of an alert and the wave arrival at shore requires an efficient and secure way to inform the community of the safe routes to shelters. This problem has been pointed out by emergency managers and the general population as one of the most important issues in previous tsunamis. Figure 6 shows the information flow from earthquake to notification. The tsunamiproducing earthquake is detected in the ocean floor. The EOC issues an alert through every means available. However, as illustrated in the figure, if there is a general blackout or a collapse in the communications, access to Internet would be jeopardized. In this case, the alternative network based on the WU proposed in this paper keeps the information flow active in both 


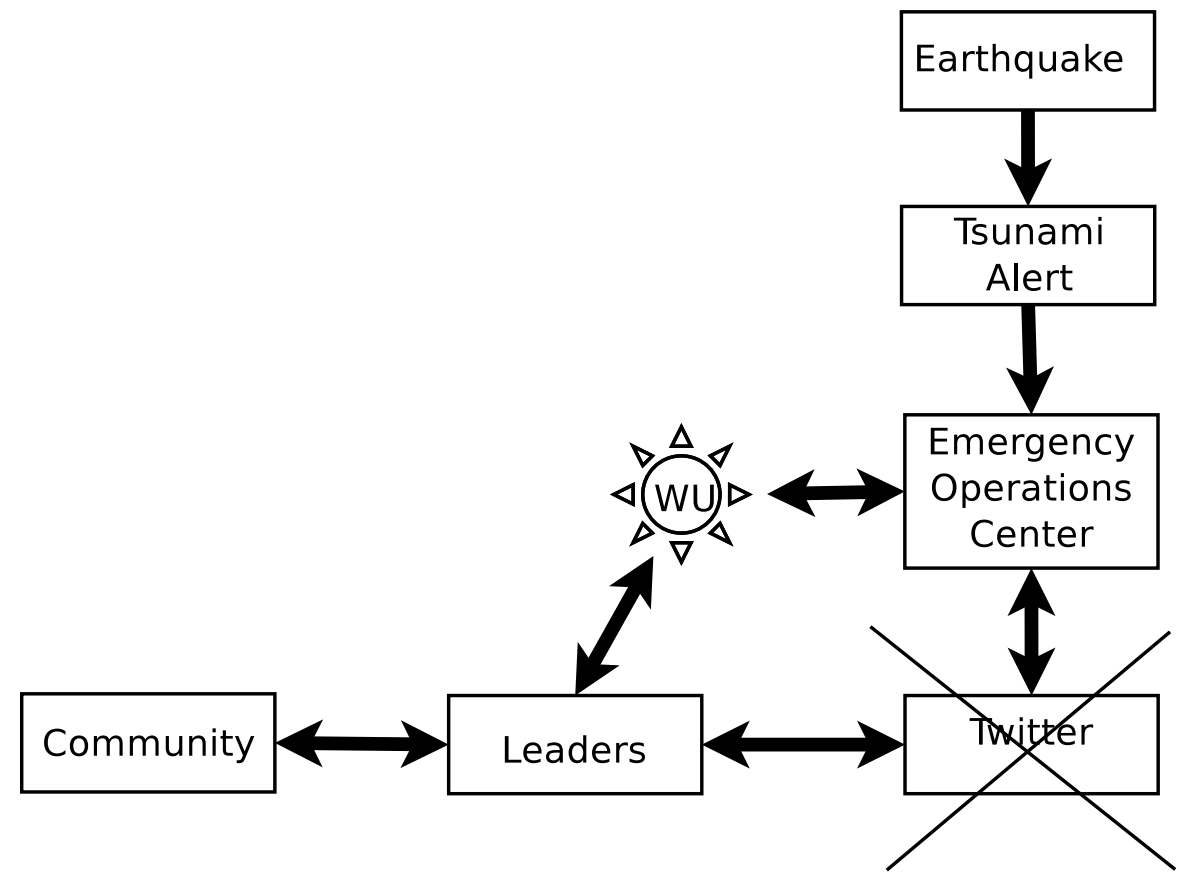

Figure 6: Information Flow in the System

directions between the EOC and the community. These devices are deployed in such a way that people moving towards the shelters are continuously in contact with the EOC. The interaction follows a pervasive paradigm. That is, the use of the technology is transparent and users are not aware of how the information is being received and forwarded. It is also ubiquitous as the deployment guarantees that the distance between two consecutive WUs is short enough.

\section{Extended Functionality}

The deployment of our WUs in the city has an additional benefit: the computational power of the processor, the continuous solar recharging, and the open hardware architecture on which the $\mathrm{WU}$ is built allow the incorporation of other types of sensors such as accelerometers, gas detectors, thermometers, sound detectors, among others. In this way, the WU can be seen as an opportunistic wireless sensor network, providing some real-time information about the area where WUs are deployed. The intermittency of the sensor network is due battery/energy: there is a minimum charge that would 
be needed for the emergency situation, and the WU would not function as a sensor network below that threshold.

If the WU does not respond to the requests of the EOC, this would be a clear indication of the severity of the damage in the area (during a confirmed disaster situation) or that the WU needs to be serviced (during normal operation).

The WUs are also useful after the disaster/event, if search and rescue teams return to the affected areas looking for survivors and victims. Furthermore, the WUs can be deployed as multi-sensors to be used during normal daily life, such as traffic cameras and up-to-date traffic information and incidents, to name only two common-case uses.

These multiple purposes can clearly make the system more affordable (cost per unit of information) due to the extra functionality that can be shared with different city offices who would contribute toward the WU network deployment.

\section{Related Work}

The use of opportunistic networks for disaster relief management has been proposed before. In (Martín-Campillo et al., 2011), the authors introduce two applications based on smartphones to transfer information. The system uses RFID and determines coordination points where information may be finally transferred to a regular network. However, the authors do not explain how the bridge to the regular network is done. In (Ochoa and Santos, 2015), the utilization of a Human Wireless Sensor Network is proposed to coordinate the action of first responders in natural disasters. Rescuers are at the same time sensors of what is going on and reporters that send this information to the command center (equivalent to the EOC). The proposed WU network complements the Human Wireless Sensor Network with a more detailed description of the WU architecture, deployment of WUs, and their implementation. In (Deb et al., 2012), the authors introduce a peer-to-peer framework to coordinate the actions of rescuers in the field, the command center, and the EOC. However, they only propose the opportunistic routing between smartphones or mobile devices without introducing the idea of an alternative infrastructure, like the WU network proposed here.

The generalization of social-media applications like Twitter or Facebook provides a different channel to disseminate information of an on-going event and the coordination of the evacuation process (Chitumalla et al., 2008). The 
problem with social media, as already highlighted throughout this paper, is that these channels are unlikely to work during disaster (due to power failure and network overloading). Our proposed architecture with the WU network could be used instead of and/or together with the regular network architecture on which social media relies.

\section{Conclusions}

This article presents the design of a Witness Unit (WU) to be used as an alternative communication network in early alerts for near-field tsunamis. Such tsunamis arrive at the coast within 15 to 30 minutes after an earthquake, which likely destroyed the infrastructure leading to electricity and communications black-outs. In these situations, the use of alternative networks is fundamental to guide and coordinate an evacuation process. The WU relies on the Iridium SBD system for communication and acts as a gateway to an ad-hoc Wi-Fi network to which community leaders can connect. Our WU design comes with a good amount of memory and a powerful microprocessor. Both characteristics allow the implementation of a distributed system in which WUs are autonomous and can assign people to shelters and compute the best route to them. If a cheaper version of the WUs is needed (e.g., for affordability or other reasons), our design also allows for WUs to send updated information observed by the leaders in the field, to the EOC, which in turn can send further, new, revised updates to all leaders. WUs are fundamental for the information flow between the EOC and the community leaders who will disseminate the information to the general population.

Appropriate location of WUs throughout the city is fundamental to provide this alternative communication channel. The distribution of WUs is similar to the coverage problem and has been proved to be a complex problemo (NP-hard). In this paper we have provided a greedy heuristic algorithm that solves the allocation problem quickly and effectively. WUs can also be used as a wireless sensors network and as a support network for the search and rescue teams after the disaster. These extended functionalities could attract technical and financial support from (more) public agencies.

\section{Acknowledments}

The authors thank Mark Dunn and Ai Fuli for their help in the development of this article. 
This paper was financed with National Science Foundation grant: OCE 1331463, Hazards SEES Type 2: ?From Sensors to Tweeters: A Sustainable Sociotechnical Approach for Detecting, Mitigating, and Building Resilience to Hazards.

\section{References}

Ai, F., Comfort, L. K., Znati, T., 2015, this issue. A dynamic decision support system based on geographical information and mobile social networks: A model for tsunami risk mitigation in padang, indonesia.

Bluetooth, 2014. Specification of the bluetooth core system v4.2. URL https://www.bluetooth.org/en-us/specification/adopted-specifications

Busanelli, S., Rebecchi, F., Picone, M., Iotti, N., Ferrari, G., 2013. Crossnetwork infromation dissemination in vehicular ad hoc networks (vanets): Experimental results from a smartphone-based testbed 5 (3), 398-428.

Cao, Y., Sun, Z., Second 2013. Routing in delay/disruption tolerant networks: A taxonomy, survey and challenges. Communications Surveys Tutorials, IEEE 15 (2), 654-677.

Carley, K., Malik, M., Pfeffer, J., M., K., 2015, this issue. Crowd sourcing disaster management: The complex nature of twitter usage in indonesia.

Chitumalla, P., Harris, D., Thuraisingham, B., Khan, L., Jan 2008. Emergency response applications: Dynamic plume modeling and real-time routing. Internet Computing, IEEE 12 (1), 38-44.

Coley, G., 2015. Beaglebone black system reference manual. http://beagleboard.org/BLACK.

Deb, D., Bose, S., Bandyopadhyay, S., 2012. Coordinating disaster relief operations using smart phone/pda based peer-to-peer communication. International Journal of Wireless \& Mobile Networks 4 (6).

Galluccio, L., Morabito, G., Moustakas, A., Palazzo, S., 2010. Opportunistic communications in infostation systems: Delay and stability analysis. In: Global Telecommunications Conference (GLOBECOM 2010), 2010 IEEE. pp. $1-5$. 
Hart, M., 2014. Arduino iridiumsbd library for rockblock. https://github.com/mikalhart/IridiumSBD.

Huang, C.-F., Tseng, Y.-C., 2005. The coverage problem in a wireless sensor network 10, 519-528.

Huang, C. M., Lan, K. C., Tsai, C., 2008. A survey of opportunistic networks. In: Proceedings of the 22nd International Conference on Advanced Information Networking and Applications.

IEEE, 2010. Ieee draft standard for information technology - telecommunications and information exchange between systems - local and metropolitan area networks - specific requirements - part 11: Wireless lan medium access control (mac) and physical layer (phy) specifications. IEEE Draft P802.11-REVmb/D3.0, March 2010 (Revision of IEEE Std 802.11-2007, as amended by IEEE Std 802.11k-2008, IEEE Std 802.11r-2008, IEEE Std 802.11y-2008, IEEE Std 802.11w-2009 and IEEE Std 802.11n-2009).

Intel, 2015. Arduino intelgalileo. http://arduino.cc/en/ArduinoCertified/IntelGalileo.

Iridium, 2012. Iridium Short Burst Data Service Developers Guide.

Liu, M., Yang, Y., Qin, Z., 2011. A survey of routing protocols and simulations in delay-tolerant networks 6843, 243-253.

Martín-Campillo, A., Martí, R., Yoneki, E., Crowcroft, J., 2011. Electronic triage tag and opportunistic networks in disasters. In: Proceedings of the Special Workshop on Internet and Disasters. SWID 2011. ACM, New York, NY, USA, pp. 6:1-6:10.

URL http://doi.acm.org/10.1145/2079360.2079366

Meier, P., 2015. irevolutions, social media for disaster response done right! http://irevolution.net/tag/flooding/.

Moreira, W., Milheiro Mendes, P., 2012. Opportunistic routing based on daily routines. In: IEEE International Symposium on a World of Wireles, Mobile and Multimedia Networks.

Nguyen, H. A., Giordano, S., 2009. Routing in opportunistic networks 1 (3). 
Ochoa, S. F., Santos, R., 2015. Human-centric wireless sensor networks to improve information availability during urban search and rescue activities. Information Fusion 22 (0), $71-84$.

Raspberry, 2015. Raspberry pi. http://www.raspberrypi.org/products/raspberrypi-2-model-b/.

Stankovic, J., Oct 1988. Misconceptions about real-time computing: a serious problem for next-generation systems. Computer 21 (10), 10-19.

Wybo, J., Lonka, H., 2002. Emergency management and the information society: how to improve the synergy? 1 (2).

ZigBee, 2015. Zigbee specificaction.

URL http://www.zigbee.org/zigbee-for-developers/applicationstandards/ 


\section{Appendix A.}

In this appendix the heuristic algorithm for the localization of the WU is presented in Figures A.7 and A.8. We argue that the complexity of the algorithm is $O(n m)$ where $n$ is the number of nodes and $m$ is the number of edges. To show this, we can assume, in the worst case, that every node will be incorporated into set $S$ so the whole graph will be analyzed. At the same time, every edge will have to be inspected in each case. Two simple examples are presented at the end to show the way in which the algorithm works.

Given $D$ (maximum time), $G(V, \xi)$ for region $R_{i}$ and a set of paths (evacuation routes) pre-loaded with WU located at nodes $s_{i} \in V$, we define:

a. $S=\left\{s_{i}\right\} \subseteq V$.

b. $N(v)$ is the set of neighbor nodes of $v$, they are connected through a direct edge.

c. $\operatorname{maxweight}(v, P)=\max l(u, v) \quad \forall u \in N(v) / P$, where $l(u, v)$ is the weight of the edge between nodes $u$ and $v$.

d. $L$ is the accumulated sum of weights.

e. $W$ is the set of nodes already analyzed.

Locate $(V, \xi, S, D)\{$

1. $W \leftarrow \Phi$

2. While $S / W \neq \Phi$

(a) $v \leftarrow$ node $\in S / W$

(b) $W \leftarrow W \cup\{v\}$

(c) $P \leftarrow \Phi$

(d) $L=0$

(e) Advance $(v, D, S, L, P)$

3. Endwhile

\}

Figure A.7: Algorithm to select the placement of the WU in the intersections 
Example. Figure A.9 presents a simple graph for the allocation of the WUs. For this case, $V=\{a, b, c, d, e, f, g\}$ (set of all vertices/crossings, $S \leftarrow a$ (set of vertices where a WU will be place), and $D=3.5$ (the maximum distance between WUs. By following the algorithm we have the following graph traverse: $a b, a b c, a b c d, a b c d f$, and we can see that when $L$, the cumulative distance from the previous $\mathrm{WU}$, exceeds $D$, we assign the $\mathrm{WU}$ to the last traversed crossings; that is, when $l(f, e)+L=4.6>D, S \leftarrow\{a, f\}$. After this, the algorithm continues with the other branch of $b$ : abe, abef. The next element in $S$ is $f$. As the edges between $f$ and $d$ and between $f$ and $e$ have already been considered, only $f g$ is followed. With no more nodes in $S$ to search, the final WU locations will be $a$ and $f$. In theory, the algorithm may require 49 steps (worst-case analysis), however, this solution required only 7 steps.

The example shows how the algorithm finds a solution given the predefined locations. If we changed the starting point, a different distribution of WUs would be found. For example, if the pre-allocated WU was placed in node $d$, an additional $\mathrm{WU}$ would need to be placed in $e$ to achieve the required coverage. The algorithm is greedy and may obtain non-minimal solutions.

Figure A.10 shows an example where the solution obtained by the Locate algorithm is not optimal. The initial element in set $S$ is node $a$. For a maximum distance $D=3$, the Locate algorithm finds $d$ and $f$ as nodes in which a WU should be placed. It is easy to see that placing a WU in node $c$ solves the problem. The solution provided by Locate, however, is more resilient as each $\mathrm{WU}, d$ and $f$, cover a different path. With only $c$ covering both branches, a failure in that point leaves both paths without coverage. In any case, as the allocation of WU is made considering several factors, a second optimization routine may be used to prune redundant WU if necessary. 
Advance $(v, D, S, L, P)\{$

1. If $L+$ maxlength $(v, P)>D$

(a) If $v \in S$

i. There is no solution and a new node should be inserted for a WU.

(b) Else

i. $S \leftarrow S \cup\{v\}$

(c) Endif

2. Else

(a) $P \leftarrow P \cup\{v\}$

(b) While $N(v) / S / P \neq \Phi$

i. $u \leftarrow N(v) / S / P$ such that $l u, v=\operatorname{maxweight}(v, P)$

ii. Advance $(u, D, S, L+l(u, v), P)$

(c) Endwhile

3. Endif

\}

Figure A.8: Algorithm for traversing $G(V, \xi)$

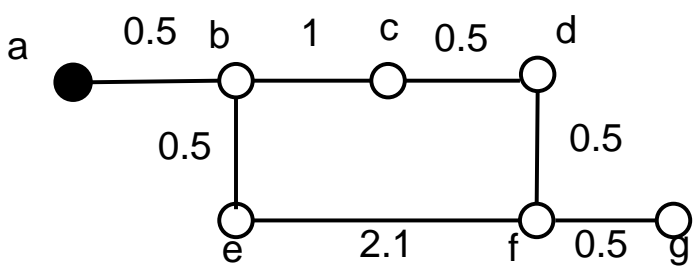

Figure A.9: WU Allocation in a simple graph 


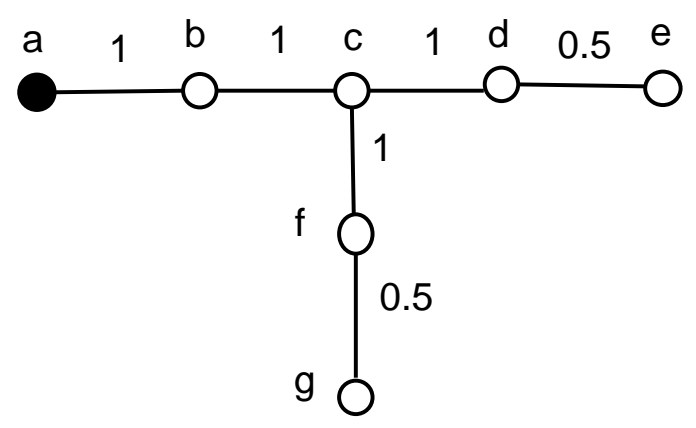

Figure A.10: Non-optimal allocation example 